\title{
Interdisciplinary challenges in the study of power grid resilience and stability and their relation to extreme weather events
}

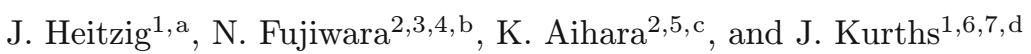 \\ 1 Potsdam Institute for Climate Impact Research, Potsdam, Germany \\ ${ }^{2}$ Institute of Industrial Science, The University of Tokyo, Tokyo 153-8505, Japan \\ 3 FIRST, Aihara Innovative Mathematical Modelling Project, Japan Science and \\ Technology Agency, The University of Tokyo, Tokyo 153-8505, Japan \\ ${ }^{4}$ Center for Spatial Information Science, The University of Tokyo, Chiba 277-8568, Japan \\ ${ }^{5}$ Graduate School of Information Science and Technology, The University of Tokyo, Tokyo \\ 113-8656, Japan \\ ${ }^{6}$ Department of Physics, Humboldt University of Berlin, Berlin, Germany \\ 7 Institute for Complex Systems and Mathematical Biology, University of Aberdeen, \\ Aberdeen, UK
}

\section{Received 3 September 2014 / Received in final form 4 September 2014} Published online 26 September 2014

\begin{abstract}
This topical issue collects contributions to the interdisciplinary study of power grid stability in face of increasing volatility of energy production and consumption due to increasing renewable energy infeed and changing climatic conditions. The individual papers focus on different aspects of this field and bring together modern achievements from various disciplines, in particular complex systems science, nonlinear data analysis, control theory, electrical engineering, and climatology. Main topics considered here are prediction and volatility of renewable infeed, modelling and theoretical analysis of power grid topology, dynamics and stability, relationships between stability and complex network topology, and improvements via topological changes or control. Impacts for the design of smart power grids are discussed in detail.
\end{abstract}

Many countries face the problem that reducing greenhouse gas emissions while phasing out nuclear energy requires a transformation of the energy system that includes large amounts of renewable energy production. The latter, however, is highly influenced by extreme weather events, which are likely to get more frequent and more severe due to climate change. Extreme events influence not only the availability of energy, but also and of more fundamental importance, the stability of the whole power grid, which is not yet understood sufficiently. Grid operation and control will profit

\footnotetext{
a e-mail: heitzig@pik-potsdam.de

b e-mail: fujiwara@csis.u-tokyo.ac.jp

c e-mail: aihara@sat.t.u-tokyo.ac.jp

d e-mail: kurths@pik-potsdam.de
} 
from cutting-edge methods from physics, control theory, networks of coupled nonlinear oscillators and advanced nonlinear data analysis.

At the same time, a thorough investigation of the interrelationship between extreme events, grid stability, and the consequences of power outages during extreme events under conditions of climate change and growing renewable energy production, and the question of how to design a resilient grid will profit from the viewpoint that considers interactions and correlations in the network structure of the global power grid, the global climate/weather system, and potentially other networks such as communication networks and socio-economic systems.

While the engineering community has developed quite detailed and highly efficient power system models but has mainly employed linearisation-based methods and complicated simulations to analyse their stability, the physics community typically uses more simplified models but has recently developed more advanced concepts and methods including nonlinear data analysis and complex network theory, which are promising also in the power grid context.

To foster these synergetic effects between the disciplines, we have collected original research articles and reviews from the physical, engineering, mathematical, and geo-science fields that relate to the thematic complex of resilient power grids and extreme events.

This special issue on Resilient Power Grids and Extreme Events consists of 20 original papers covering various aspects and recent achievements of the interdisciplinary study of power grid stability in face of increasing volatility of energy production and consumption due to increasing renewable energy infeed and changing climatic conditions. These papers can be roughly grouped into five categories, namely, surveys [1-4] prediction and volatility of renewable infeed [5-7] derivation of requirements for a resilient power grid infrastructure [8-13] relationships between grid stability and complex network topology [14-17] and the analysis of special issues in dynamic stability [20-22]. Because of the various overlaps, we still chose to assemble these works in alphabetical order in this volume.

In the opening tutorial review [1], the topic is introduced by discussing a recent major power blackout in Europe, before an overview of the electrical engineering state of the art in the study of transient and steady-state stability of traditional power grids by means of electromechanical dynamical models is given, with a focus on determining regions of attraction and the possibility of stability improvement via feed-back control.

The theory overview is complemented from the physics perspective in the review [2] on networks of the diffusively coupled complex Ginzburg-Landau oscillators, which describes the limit-cycle oscillation near the supercritical Hopf bifurcation point. In particular, how the synchronised state destabilises through the Benjamin-Feir instability and emerging spatial patters are illustrated. The stability of power grids can be viewed as synchronisation of limit cycle oscillators on complex networks, so this review gives us insights how to tackle this problem.

The review [3] focusses on stability issues arising in the emergent non-traditional power grids with additional smart grid components that necessitate broader interdisciplinary models incorporating large amounts of renewable energy production, information networks, and producer and consumer behaviour, using cutting-edge techniques such as complex network analysis and multiagent simulation.

Completing the review section, [4] uses an analysis of a case study of a major power outage in East Asia caused by an extreme weather event to set the technological issues into the broader context of risk management including climate, economic, and political issues to illustrate the need for a complex systems view of power grids and their socioecological framework. 
The first group of original research articles focusses on the energy production side, mainly dealing with the prediction and volatility analysis of solar and wind power infeed. In [5] a novel method for predicting a multivariate time series with confidence bounds is developed and tested with spatially distributed hourly averaged solar irradiation and wind speed data, showing promising results for 36 hours ahead. The study [6] uses a state of the art meteorological model on a $15 \mathrm{~min}$ time interval to show that since aerosols can cause extreme solar irradiation events, accurate solar energy day-ahead production forecasts rely on good aerosol predictions. Finally, [7] is concerned with fluctuations on the shorter time-scales of seconds to minutes relevant for transient grid stability, revealing a turbulent-like power-law behaviour of the spectrum of both solar and wind power data from several locations, with solar being particularly intermittent.

Combining insights about renewable production and power grid dynamics, the second group of research papers derives requirements for a resilient power grid infrastructure and discusses possible components such as control and storage. In [8] the consequences of the ambitious plans of the German "Energiewende" for extending the transmission grid to cope with huge shares of renewable production are analysed by means of a new combination of a power grid model, an energy system model and a unit commitment model representing production decisions. The study shows that the planned extensions are likely sufficient to avoid congestions and overloads in plausible future production scenarios even in the single-line outage case. The authors of [9] study the frequency fluctuations caused by volatile wind power in the presence of load frequency control systems that compensate fluctuations dynamically using thermal power plants. Their new analysis method indicates that these thermal plants' capacity is more important than their responsiveness. How load frequency control may be improved using information about neighbouring generators by means of hierarchically distributed stabilising controllers is the topic of [10]. Dealing with longer-term fluctuations and temporary supply-demand mismatches, the paper [11] identifies a phase transition in the required amount of storage in highly renewable electricity systems, showing that for cost-efficient reasons renewable penetration should be either well below $100 \%$ or, perhaps surprisingly, slightly larger than $100 \%$. The special form of storage via electric car batteries is analysed in [12], a paper in which a centralised strategy for scheduling charging patterns is proposed. Finally, [13] discuss optimal power transmission and distribution in so-called Digital Grids that are segmented into synchronised parts which are connected asynchronously, an emerging technology that can improve grid stability and controllability.

Another group of papers deals with the complex network topology of power grids and its relationship to grid stability. The random network generation model in [14] is designed to produce synthetic ensembles of power grid topologies with realistic statistical properties, to compensate for the scarcity of real-world grid topology data freely available for research. It is based on an initial minimal spanning tree and successive growth using a cost-redundancy tradeoff and optional line-splitting. In $[15,16]$, the recent nonlinear stability concept of basin stability [16] is used to exhaustively study all two-generators-two-consumers topologies.

In [17], another nonlinear stability concept is used to assess robustness under variation of effective power in load nodes, finding that in a case study of the eastern Japanese grid robustness is highly correlated with shortest path length from generators, and demonstrating that reactive power enhances robustness. Finally, [18] is an analysis of the effect the switching on an additional transmission line in a larger grid has on the power flow distribution, revealing a slow decay of the resulting perturbation, an instance of the Braess paradox [19], and a somewhat counterintuitive finding that stability may decrease with a more heterogeneous distribution of consumers and producers. 
Finally, some special aspects of dynamical stability are studied in the last group of works. Focussing on real-time operational decisions in real-world power grids, [20] presents a set of security indices regarding rotor angle, voltage, and frequency stability, damping of power swings, and frequency or voltage excursions across specified thresholds, which are useful for evaluating the severity of several possible contingencies in a power system. Rotor angle and voltage stability are studied more theoretically in [21] by augmenting the widely used simple swing-equation with dynamical voltage equations and assessing basin stability in the resulting model. The work of [22] complements these studies by focussing on collective instabilities that are not related to overloads and might e.g. occur in consequence of adding additional transmission lines.

As is clear from the contributions of this special issue, there are many aspects of problems in the stability and design of power grids with renewable energy that are beyond conventional research disciplines. Hence, we hope that this collected knowledge and synergy of different fields will stimulate further research on these very challenging problems.

\section{References}

1. Gajduk, et al., Eur. Phys. J. Special Topics 223(12), 2387 (2014)

2. Nakao, Eur. Phys. J. Special Topics 223(12), 2411 (2014)

3. Nardelli, et al., Eur. Phys. J. Special Topics 223(12), 2423 (2014)

4. Ye, Eur. Phys. J. Special Topics 223(12), 2439 (2014)

5. Hirata, et al., Eur. Phys. J. Special Topics 223(12), 2451 (2014)

6. Shimose, et al., Eur. Phys. J. Special Topics 223(12), 2621 (2014)

7. Tabar, et al., Eur. Phys. J. Special Topics 223(12), 2637 (2014)

8. Pesch, et al., Eur. Phys. J. Special Topics 223(12), 2561 (2014)

9. Kashima, et al., Eur. Phys. J. Special Topics 223(12), 2493 (2014)

10. Ishizaki, et al., Eur. Phys. J. Special Topics 223(12), 2461 (2014)

11. Jensen, et al., Eur. Phys. J. Special Topics 223(12), 2475 (2014)

12. Suzuki, et al., Eur. Phys. J. Special Topics 223(12), 2631 (2014)

13. Shibano, et al., Eur. Phys. J. Special Topics 223(12), 2611 (2014)

14. Schultz, et al., Eur. Phys. J. Special Topics 223(12), 2593 (2014)

15. Ji, et al., Eur. Phys. J. Special Topics 223(12), 2483 (2014)

16. P. Menck, J. Heitzig, J. Kurths, H.J. Schellnhuber, Nat. Commun. 5, 3969 (2014)

17. Nagata, et al., Eur. Phys. J. Special Topics 223(12), 2549 (2014)

18. Labavić, et al., Eur. Phys. J. Special Topics 223(12), 2517 (2014)

19. M. Rohden, A. Sorge, M. Timme, D. Witthaut, Phys. Rev. Lett. 109, 064101 (2012)

20. Kerin, et al., Eur. Phys. J. Special Topics 223(12), 2503 (2014)

21. Schmietendorf, et al., Eur. Phys. J. Special Topics 223(12), 2577 (2014)

22. Manik, et al., Eur. Phys. J. Special Topics 223(12), 2527 (2014) 